\title{
BMJ Open Perceptions on undertaking regular asymptomatic self-testing for COVID-19 using lateral flow tests: a qualitative study of university students and staff
}

\author{
Marta Wanat (1) , ${ }^{1}$ Mary Logan, ${ }^{1,2}$ Jennifer A Hirst (D) , ${ }^{1,2}$ Charles Vicary, ${ }^{1}$ \\ Joseph J Lee, ${ }^{1}$ Rafael Perera (1) , , ${ }^{1,2}$ Irene Tracey, ${ }^{3,4}$ Gordon Duff, ${ }^{5}$ Peter Tufano, ${ }^{6}$ \\ Thomas Fanshawe, ${ }^{1}$ Lazaro Mwandigha, ${ }^{1}$ Brian D Nicholson (iD) ${ }^{1}$ \\ Sarah Tonkin-Crine (D) , ${ }^{1,7}$ Richard Hobbs ${ }^{1,2}$
}

To cite: Wanat M, Logan M, Hirst JA, et al. Perceptions on undertaking regular asymptomatic self-testing for COVID-19 using lateral flow tests: a qualitative study of university students and staff. BMJ Open 2021;11:e053850. doi:10.1136/ bmjopen-2021-053850

- Prepublication history and additional supplemental material for this paper are available online. To view these files, please visit the journal online (http://dx.doi.org/10.1136/ bmjopen-2021-053850).

ST-C and RH are joint senior authors.

Received 26 May 2021 Accepted 10 August 2021

Check for updates

(C) Author(s) (or their employer(s)) 2021. Re-use permitted under CC BY-NC. No commercial re-use. See rights and permissions. Published by BMJ.

For numbered affiliations see end of article.

Correspondence to

Dr Marta Wanat;

marta.wanat@phc.ox.ac.uk

\section{ABSTRACT}

Objectives Successful implementation of asymptomatic testing programmes using lateral flow tests (LFTs) depends on several factors, including feasibility, acceptability and how people act on test results. We aimed to examine experiences of university students and staff of regular asymptomatic self-testing using LFTs, and their subsequent behaviours.

Design and setting A qualitative study using semistructured remote interviews and qualitative survey responses, which were analysed thematically.

Participants People who were participating in weekly testing feasibility study, between October 2020 and January 2021, at the University of Oxford.

Results We interviewed 18 and surveyed 214 participants. Participants were motivated to regularly selftest as they wanted to know whether or not they were infected with SARS-CoV-2. Most reported that a negative test result did not change their behaviour, but it did provide them with reassurance to engage with permitted activities. In contrast, some participants reported making decisions about visiting other people because they felt reassured by a negative test result. Participants valued the training but some still doubted their ability to carry out the test. Participants were concerned about safety of attending test sites with lots of people and reported home testing was most convenient.

Conclusions Clear messages highlighting the benefits of regular testing for family, friends and society in identifying asymptomatic cases are needed. This should be coupled with transparent communication about the accuracy of LFTs and how to act on either a positive or negative result. Concerns about safety, convenience of testing and ability to do tests need to be addressed to ensure successful scaling up of asymptomatic testing.

\section{INTRODUCTION}

In January 2020, the WHO declared COVID-19 to be a global healthcare emergency, ${ }^{1}$ and since June 2021, the number of cases globally has now exceeded 177 million. $^{2}$ In response to this crisis, countries across the

\section{Strengths and limitations of this study}

- A unique study exploring people's experiences of regular, weekly asymptomatic self-testing including views and understanding of the accuracy of lateral flow tests, highlighting a number of barriers and facilitators to successful scaling up of asymptomatic testing.

- The mean number of tests conducted by each interview and survey participant was higher than the mean number of tests in non-interviewed participants (ie, the main study participants), so our sample may over-represent those who continued to test regularly.

- Data represented the views of staff and university students who agreed to take part, and their motivation to participate and perceived benefits of testing may be different from those in the wider university population and other non-university settings.

- The study did not capture the views of participants who had not completed any tests, which should be explored in future studies.

- The study had a relatively low response rate for both interviews and surveys and thus may not have captured the views of all participants (eg, those who disengaged with the study and, subsequently, testing).

world have implemented numerous strategies to tackle the pandemic, including various grades of restriction in population movement, social distancing, use of face coverings in public and testing for patients with typical symptoms using laboratory COVID-19 PCR tests. While these tests are considered the gold standard for diagnosis, they have limitations, including slow turnaround time, specialist facilities needed for processing, and detection of non-infectious SARS-CoV-2 particles, limited access and high costs. ${ }^{3}$

Some individuals infected with SARS-CoV-2 remain asymptomatic, and in symptomatic 
cases, viral shedding is likely to occur before symptoms occur. ${ }^{4}$ Consequently, recent data showing that one in three people infected with SARS-CoV-2 may not show symptoms prompted an increased interest from governments in the feasibility of testing asymptomatic individuals using lateral flow tests (LFTs) ${ }^{5}$ In fact, in a number of countries,${ }^{67}$ including the UK, ${ }^{8}$ these devices have been part of government strategies for easing of lockdowns.

LFTs were brought to market for the rapid detection of SARS-CoV-2 antigen in late $2020 .{ }^{9}$ LFTs do not require specialist laboratory equipment and can be performed in the community with results returned within $30 \mathrm{~min}$. In October 2020, the UK government launched a masstesting initiative using LFTs across the UK. ${ }^{10}$ However, the diagnostic performance of one-off LFTs ${ }^{11}$ does not meet the WHO accepted minimum of $97 \%$ specificity and $80 \%$ sensitivity, and there is still limited evidence related to diagnostic performance when used by individuals without symptoms. ${ }^{12}$ In order to deliver a successful testing programme, it was proposed that testing needs a $90 \%$ or higher uptake followed by isolation of infected individuals, ${ }^{13} 14$ coupled with retesting every few days to improve the diagnostic performance of the overall testing strategy. ${ }^{15}$ In the UK, the LFTs are authorised for use in detecting positive cases of asymptomatic individuals and are now used in schools and before larger events. The public can also order two cost-free tests per week for general use. ${ }^{16}$

Asymptomatic testing (screening) has attracted a lot of attention, with many highlighting that LFTs can give people false reassurance ${ }^{17}$ and others stressing that targeted testing could help reduce transmission. ${ }^{18} 19$ As there are no clinical trials of SARS-CoV-2 screening, there is uncertainty of the effect. The supposition is that testing frequently may identify asymptomatic cases willing to self-isolate, which could reduce transmission of infections more than any increase in transmission that arises from falsely reassuring people with falsenegative results. For any given test, people's behaviours related to use of tests and behaviours following the test will determine this balance. Screening pilots usually request that participants do not change their behaviour as a result of being tested and receiving a negative result. The evidence from point-of-care tests for other conditions is that tests are part of complex interventions that change behaviours in unpredictable ways. ${ }^{20}$ Evidence is therefore needed on end-user perceptions on the use of COVID-19 LFTs.

Few studies have examined acceptability and feasibility of asymptomatic testing in the community ${ }^{21}$ using PCR tests, ${ }^{22} 23$ and even fewer have focused on these issues in relation to regular testing (rather than one-off) ${ }^{23}$ and LFTs. People's views and understanding of the accuracy of such tests have also not been explored. Our study aimed to address this gap by examining experiences of university students and staff of weekly self-testing using LFTs with the aim of identifying key lessons for future asymptomatic testing programmes.

\section{METHODS}

\section{Study design}

This is a qualitative study embedded within a mixedmethods cohort study.

\section{Study recruitment}

The Feasibility and Acceptability of community COVID-19 Testing Strategies (FACTS) study was a mixed-methods cohort study conducted at the University of Oxford. The recruitment began in two sites on 29 October and in one site on 17 November 2020 (sites A, B and C). In late November, recruitment was extended across the University but stopped in early December when a Universitywide COVID-19 screening programme was implemented leaving only sites A, B and C continuing with the study. Invitations to participate were sent by email, first only to students and later to staff, to take part in once-a-week testing. The email invitation also contained a 5 min video explaining how to prepare to test, perform the swab, extract the sample, test the sample and read the results. Eligible participants were asked to complete an e-consent process on the 'CVm-Health +Education' app on their phones, which was developed by Sensyne Health PLC (Oxford, UK). Those who wanted further information had the opportunity to ask the research team members questions at the training session and then consent via the app if they were happy to participate.

\section{Sampling}

Eligibility criteria for the main study included age $>16$ years old, working or studying across the chosen sites at the University, showing no COVID-19 symptoms (new continuous cough and/or high temperature and/or loss of/change to, sense of smell/taste) and being capable of LFT self-administration following training.

\section{Training}

The email invitation also contained a 5 min video explaining how to prepare to test, perform the swab, extract the sample, test the sample and read the results. Participants were invited to a training session conducted face-to-face or online, before undertaking weekly testing. Face-to-face training was carried out at participants' work or place of study. Participants were talked through the aims of the study, followed by a demonstration of the swabbing, sample preparation, testing, and recording and interpreting the test result. Following the demonstration, participants performed the test, with support from the trainers if required. When $30 \mathrm{~min}$ had elapsed since applying the sample to the test device, participants were asked to interpret the result, record this on the app and photograph the result using the app. The trainers visually checked each test result and confirmed whether the participant's interpretation of the result was correct or not. For a small number of participants, live online training was offered. These participants collected test kits in advance from a central location. This online training was delivered by two trainers via Zoom and involved similar 
procedures to the face-to-face training. The session was interactive so participants could ask questions. Everyone had also been provided with PDF step-by-step illustrated guide to testing.

The participant information sheet informed participants that rapid tests are not as reliable as, or a replacement for, a PCR test. In the event of a positive test result, participants were advised to self-isolate and book a PCR test through the University COVID-19 testing service; in the event of a negative test, participants were advised to follow normal infection prevention procedures. This information was repeated during the training where participants were also told that there is limited evidence on the accuracy of the test in asymptomatic people. At the time of the study, the Innova test had not received MHRA authorisation for asymptomatic testing nor for self-testing, and the test was used under research ethics.

\section{Testing processes}

While the original plan was to provide all participants with the testing kits to take home after training, this was not possible in the initial stages of the study. This meant that the repeat weekly testing took place at the test sites until late November. This involved participants attending at a testing station at their college or department. Thereafter, participants recruited to the three main sites (sites A, B and $\mathrm{C}$ ) were supplied with sufficient test kits to continue testing in their accommodation or home throughout the Christmas break until 18 January 2021 (the study end date). Follow-up did not take place at the other recruiting sites. If participants missed administering a test, they were still eligible to remain in the study and resume testing on a weekly basis. If a participant received a COVID-19 positive result from a PCR test, they were asked to stop self-testing. Participants could withdraw from the study by contacting the study team without providing a reason.

All participants used the same test, the Innova Rapid SARS-CoV-2 Antigen Test Kit (Innova Medical Group, USA), developed for testing symptomatic individuals. The manufacturers report it can detect the SARS-CoV-2 antigens within $20 \mathrm{~min}$ and a negative test result within 30 min. ${ }^{24} 25$ The instructions for use require nasal and tonsil swabbing, transferring of the sample to an extraction buffer solution then application of the mixture to the device. $^{26}$

\section{Interview recruitment and data collection}

Eligibility criteria for the interview study included age $>16$ years old, working or studying across the chosen sites at the University. A selection of participants who agreed to be contacted about an interview at the study enrolment stage were invited via email. We used purposive sampling in order to obtain variation in university role (student or staff) and department, aiming to obtain an equal number of staff and student participants. A semistructured interview guide was developed based on the primary research questions (online supplemental appendix 1). Participants were asked about their views and experiences of using the tests, their reasons for taking part in the study, barriers and facilitators to undertaking regular testing, trust in test results and intentions to act on a positive result. After explaining the purpose of the interview, participants were asked to give oral consent, which was in addition to the main study consent. Following the interview, they were also provided with a written record of their consent. The interviews were conducted by a senior female postdoctoral qualitative researcher (MW) over the telephone or using videoconference software, depending on participant preference. The interviewer was not involved in the training of participants. The interviews were audio recorded and continued until data indicated saturation. ${ }^{27}$

\section{Survey recruitment and data collection}

As part of the wider study, we also conducted a survey examining participants' views of regular testing (online supplemental appendix 2). The survey was designed using JISC Online Surveys software, which was in line with Information Governance procedures of the University of Oxford. The survey was sent to all participants of the main study via email, asking them to complete it as part of the study. There was no separate consent form for the survey as it was part of the main study, to which all participants gave consent via the app. After data collection was completed, the data were deleted from the platform and stored securely on a University network.

\section{Data analysis}

Based on the free text comments from the survey, MW and ST-C created an initial framework consisting of nine categories that captured key areas of interest. Using this framework, detailed summaries of interview data, including verbatim quotes, were made directly from the audio recording after each interview. ${ }^{28}$ These were further developed and then used to create themes and subthemes. This method is deemed a pragmatic and efficient approach to collect and analyse data rapidly during a public health emergency. ${ }^{29}$ NVivo V.12 was used to facilitate data analysis.

\section{Patient and public involvement (PPI)}

This study was rapidly set up and therefore did not include any PPI input. All participants will receive a summary of the results.

\section{RESULTS}

At the three primary recruitment sites (A, B and C), 551 participated. A further 183 participants were recruited across other sites, so the total sample size was 734 . Between October 2020 and January 2021, they performed 3187 LFTs.

Participants completed a mean of 4.3 tests over a mean of 4.8 weeks. Eighty per cent $(n=588)$ of the participants were students, with a mean $\pm \mathrm{SD}$ age of $26 \pm 8$ years; $20 \%(\mathrm{n}=146)$ were staff, with a mean \pm SD age of $42 \pm 11$ years. Overall, $55 \%$ were women and $83 \%$ were of white ethnicity. Table 1 describes demographic characteristics of all FACTS study participants per site. 
Table 1 Demographic characteristics of participants in the main study

\begin{tabular}{lllllll}
\hline & \multicolumn{5}{c}{ Three main sites } & \multicolumn{1}{c}{ Other sites } \\
\cline { 2 - 5 } & Overall & $\begin{array}{l}\text { Combined main } \\
\text { sites }\end{array}$ & Site A & Site B & Site C & O \\
\cline { 2 - 5 } Participants, N (\%) & 734 & 551 & $165(20)$ & $141(18)$ & $245(50)$ & 183 \\
\multicolumn{1}{c}{ Staff } & 146 & 115 & 31 & 23 & 61 & 31 \\
$\quad$ Students & 588 & 436 & 134 & 118 & 184 & 152 \\
Number of tests & 3187 & 2728 & 1047 & 690 & 991 & 459 \\
Male sex, N (\%) & $327(45)$ & $244(44)$ & $64(39)$ & $56(40)$ & $124(51)$ & $83(45)$ \\
Mean (SD) age & $28.8(10.7)$ & $29.3(10.7)$ & $27.4(12.0)$ & $25.4(10.4)$ & $32.7(8.6)$ & $27.5(10.7)$ \\
White ethnicity, N (\%) & $608(83)$ & $451(82)$ & $150(91)$ & $131(93)$ & $170(69)$ & $157(86)$ \\
\hline
\end{tabular}

Of 734 participants, 431 (59\%) indicated on the main study consent form that they would be interested in taking part in an interview. Fifty-two were approached and 18 participants responded, and all chose to take part (response rate $35 \%$ ). Of these, 3 were undergraduate students; 3 were postgraduate students; and 12 were staff. Each interview participant had completed between 3 and 10 tests during the whole study period (mean 7.7). Table 2 provides demographic characteristics of interview participants. The interviews took place between 11 December 2020 and 18 January 2021 and lasted between 17 and 43 min (mean $26 \mathrm{~min}$ ). In addition, 214 participants completed the survey (29\%); 62 provided additional free text comments. Each survey participant completed between 1 and 13 tests (mean=5.8). Table 3 provides demographic characteristics of the survey participants.

We identified four themes in relation to participants' views and experiences on SARS-CoV-2 self-testing with LFT, which we report below with supporting quotes.

\section{Theme 1: perceived benefits to regular testing}

Participants reported three main benefits of taking part in the study and having access to regular self-testing. First, they wanted to check regularly whether or not they were infected with SARS-CoV-2, to reduce their fear of unknowingly infecting others, which was a concern they mentioned frequently. In addition, participants wanted to know if they were infected so they could take appropriate action, that is, self-isolate and thus minimise the risk of spreading the virus.

Second, some students highlighted that deciding to self-test with an LFT was perceived as a personal choice and was therefore more acceptable than undertaking National Health Service (NHS) or university testing when experiencing symptoms. The university protocol for symptomatic testing required everyone in the household to enter into isolation at the time of getting a test rather than at the time of getting a positive result. As participants explained, peer pressure may prevent people from doing NHS or university testing.

Getting an NHS test is such an ordeal and in a university context, there is pressure not to get tested because getting that test puts your whole house into a lockdown. This test removes barriers [...] You do it as a personal choice and not something where you get permission from the whole household to get tested (P2, student, interview)

Finally, all interviewees wanted to support COVID-19 research to contribute to fighting the pandemic.

\section{Theme 2: perceptions of test accuracy and its implications}

Overall, participants mostly accepted that tests were not $100 \%$ accurate. This was related to the fact that they saw them as just one of the measures to try to avoid spreading the virus (among social distancing, face masks and future vaccines). Some participants lacked any recalled information on test accuracy, while others sought their own information on the accuracy of LFTs in general or had heard information from family and friends. The perceived accuracy varied greatly, with participants citing figures between $50 \%$ and $90 \%$. It is important to highlight that often the same figure was seen as reassuring by some participants and not by others.

Table 2 Demographic characteristics of interview participants

\begin{tabular}{lccccc} 
& Overall number & Site A & Site B & Site C & Other sites \\
\hline Participants, N & 18 & 8 & 3 & 4 & 3 \\
Role: staff, N & 10 & 4 & 3 & 2 & 1 \\
Students, N & 8 & 4 & 0 & 2 & 2 \\
Male sex, N (\%) & $11(61)$ & 4 & 2 & 4 & 1 \\
Mean (SD) age & $35.1(14.2)$ & $34.6(17.3)$ & $45.3(10.8)$ & $28.8(6.80)$ & $34.3(15.3)$ \\
White ethnicity, N (\%) & $17(94)$ & $7(88)$ & $3(100)$ & $4(100)$ & $3(100)$ \\
\hline
\end{tabular}


Table 3 Demographic characteristics of survey participants

\begin{tabular}{|c|c|c|c|c|c|}
\hline & Overall number & Site A & Site B & Site C & Other sites \\
\hline Participants, N (\%) & 214 & 64 & 47 & 39 & 64 \\
\hline Role: staff, N & 72 & 19 & 21 & 16 & 16 \\
\hline Male sex, N (\%) & $101(47)$ & 22 & 31 & 14 & 34 \\
\hline Age, mean (SD) & $31.6(12.8)$ & $29.9(12.7)$ & $36.2(11.8)$ & $32.3(14.4)$ & $29.6(11.9)$ \\
\hline
\end{tabular}

I am sceptical because someone who works in the industry told me that some hospital stopped using the tests because with poor training it has an effectiveness rate of $50 \%$ (P19, student, interview)

I talked to a friend who is a nurse; and she said that they are around $60 \%$ which is a decent percentage to be accurate (P18, staff, interview)

Participants' views on test accuracy were important when making decisions about their behaviour. Participants did not view a negative test result as permission to break government guidelines but reported that negative tests increased their confidence to engage with activities that were allowed.

I am not sure [the test result] changed our behaviour but it reassured us that I am going to have two tests during that time and if they are both negative that gives you a bit of reassurance that this is a reasonable thing to do (P11, staff, interview)

Crucially, some participants did make decisions, based on negative test results, about engaging with activities where there was potential for transmission (eg, seeing a relative or extent of contact with relatives at home) because they were unaware that tests were not $100 \%$ accurate. When later learning that tests were not $100 \%$ accurate, participants were concerned about their decisions.

I have read online about the reliability of the tests and initially that gave me a lot of confidence. [...] and when I had a negative test I felt that I could go and have a cup of tea with a relative and then I read that the reliability was $[\ldots]$ so some of the figures were down in the 50 s or 60 s, so $60 \%$ and then you thought 'oh this is not that reassuring (P14, staff, interview)

I have done all these tests which were negative and after the 3rd test I was less careful for sure (P5, student, interview)

Finally, some participants were unsure whether the information they had read about LFTs was relevant to the test they had been using. They highlighted the difficulty of making a decision on whether to engage with certain (allowed) activities or not.

I read in the newspapers that when done by trained medical staff the tests are only $75 \%$ accurate, and by non-medical staff 50\%. [...] So if I have 50-50 success rate is that a good thing or is it better not to know (P3, staff, interview)

\section{Theme 3: extent of confidence in ability to do the tests}

The majority of participants felt that the training they had received enabled them to feel confident about doing the tests. While doing tests repeatedly increased participants' confidence, a number of participants were still unsure whether they were doing the test correctly, especially the tonsil swab. Some questioned whether an incorrect swab would make the test less reliable.

I have very strong gag reflex so I am unable to reach my tonsils. [...] I have not been able to get an answer on whether it is important to swab the tonsils (P8, staff, interview)

Participants felt that doing the tests at home was easier as they had access to mirrors; in contrast, they highlighted having to rely on their phone cameras to do the test on site. When doing tests at home, having a card which summarised the instructions was also suggested, as instead participants had to rewatch the video every time they were unsure about some aspect of the self-testing. In contrast, doing tests on site was perceived as helpful by some participants as they could ask other participants for tips. Seeing other people experience physical sensations such as watering eyes or gagging was helpful in knowing what to expect and provide reassurance that they 'were doing it right'.

\section{Theme 4: barriers and facilitators to regular testing}

All interviewees experienced swabbing as uncomfortable, at least to a certain extent, with some reporting having a strong gag reflex and testing causing sneezing or watering eyes. However, most participants highlighted that these sensations were temporary, manageable and were a 'small price to pay' for getting reassurance on whether they were infected (as described in theme 1).

Participants who were able to take a number of testing kits home seemed to see testing as relatively easy-to-fit tests into their weekly routine. In contrast, for participants who did not get packs to take home and who had to go to their department to self-test, it was an inconvenience and caused frustration, especially as testing took place over several weeks. This was especially the case for staff.

While training in a group was perceived as beneficial (as described in theme 2), some participants were also 
concerned about the safety of getting tested on site, related to being around other people, especially if they had not been going out much.

It was quite frustrating that we were expected to conduct the test in person in a lecture hall with many other students for the first few weeks, as this was the biggest personal risk I took in terms of COVID-19 exposure (P10, student, survey)

Going to the college [...] makes me a bit worried about exposure to COVID while testing, despite social distancing measures (P17, student, survey)

Finally, participants stated that they aimed to carry out testing on a weekly basis but wanted a reminder to do so when the test was due each week, and some felt this could provide additional encouragement.

\section{DISCUSSION}

We found that interviewees were motivated to conduct once-a-week testing as they wanted to know whether or not they were infected with SARS-CoV-2. While most participants accepted that the test was not $100 \%$ accurate, many could not quantify this further, and estimates of test accuracy varied greatly among participants. Importantly, most reported that a negative test result did not change their behaviour, but some participants reported making decisions about contact with other people when they would not have done otherwise, because they felt reassured by a negative test result. Participants valued the training, but some individuals still doubted their ability to do the test. Participants also raised the importance of safety and convenience when attending for tests on site.

\section{Comparison with existing literature}

Participants in our study wanted to have once-a-week testing to reduce their fear of accidentally infecting their family, friends or other people in their community while also wanting to contribute to fighting the pandemic. This is in line with the Liverpool COVID-SMART study, which found that people signed up to have a test as they wanted to protect their families, friends, as well as local hospitals and NHS workers. ${ }^{21}$ Only one study in a university setting explored these issues, although involving PCR tests, and also reported similar reasons. ${ }^{22}$ Our study also highlights the importance of the perceived benefits but in the context of regular once-a-week rather than one-off testing and use of LFTs. It also suggests that asymptomatic testing using LFTs may be perceived as more accessible and acceptable for students, in comparison to NHS or university testing, which has not been identified before.

Importantly, our study found that while most participants understood that the test was 'not 100\% accurate', estimates of test accuracy varied greatly among participants. Most reported that negative test results did not change their behaviour, but it did provide them with reassurance to engage with permitted activities. However, some participants felt reassured by the test and reported making decisions involving contact with other people, when they would not have done otherwise. Previous studies have only explored these issues for antibody testing. ${ }^{30}$ The Liverpool COVID-SMART study indicated that some participants had concerns about test accuracy, ${ }^{21}$ and one study in a university setting found that $79.6 \%$ of participants were confident in the outcome of their PCR test. ${ }^{31}$

Finally, while our participants described swabbing as being uncomfortable, they felt that the perceived benefits outweighed the burden of doing the tests. Having access to a number of tests which they could do at home made it easier for participants to take part, while doing the testing on site provided an opportunity for feedback on how well participants were doing the test but magnified safety concerns. Misinformation related to perception of the risk of infection at test sites and the need to have physical contact with centre staff have been described before. ${ }^{31}$

\section{Strengths and limitations}

This first qualitative study examining views and experiences of students and staff of regular asymptomatic SARS-CoV-2 testing in a university setting using LFTs highlights a number of key issues related to acceptability and feasibility of regular testing as well as its behavioural implications. We note some limitations. First, the mean number of tests conducted by each interview and survey participant was higher than the mean number of tests in non-interviewed participants (ie, the main study participants), so our sample may over-represent those who continued to test regularly. In fact, we have not captured the views of those participants who had not completed any testing during the study period. Future studies should include the perspectives of participants who did not use the test even when provided with the opportunity and resources to do so, in order to understand the barriers to uptake and regular testing. Second, the FACTS participants were university student and staff volunteers whose motivation to participate and perceived benefits may be different from those in the wider university population and other non-university settings. Third, while the aim was to recruit equal numbers of students and staff for interviews, we interviewed more staff. This was in line with the main study, where we found a higher follow-up rate in staff than in students, suggesting that staff were more likely to be compliant to testing and remained in the study for a higher proportion of their potential follow-up time than students. Finally, the response rate of the interview and survey was relatively low, which may have meant that views of some participants have not been captured (eg, those disengaged with the programme). This may be explained by the timing of both the survey and interview study; recruitment took place very close to the Christmas break, thus possibly limiting opportunities for staff and student to commit time for these aspects of the programme.

\section{Implications for policy and practice and future research}

Our study indicates that messages highlighting the benefits for family, friends and society in identifying asymptomatic cases, contributing to fighting the pandemic and ultimately lifting lockdowns might be beneficial for encouraging regular use of LFTs. However, these need to be coupled with 
clear and transparent communication about LFT accuracy. Also, given that the same reported accuracy of the test might be perceived by different people as more or less favourable, it is crucial that this is framed within clear messages on what it means for an individual's behaviour (ie, the need to follow COVID-19 safety measures). This is especially important for testing in workplaces or schools where a negative test may allow people to return to their study or workplace and will consequently involve contact with other people. Advice that supports people to continue physical distancing, hand hygiene and mask wearing in the context of a negative test is crucial. A recent report of implementation of asymptomatic testing in local authorities in England showed that 47\% of local authority websites did not explain the limitations of LFTs ${ }^{17}$ or that people should continue following safety measures despite a negative result and highlighted a lack of standard messaging on test accuracy. ${ }^{17}$

When scaling up regular asymptomatic testing, it is important to also consider potential concerns about convenience of testing, and people's confidence and ability to do the testing. In settings where people may be tested on site, safety and convenience may be important to consider. Concerns about physical sensations also need to be addressed. However, for those who are sent tests to take at home, clear information on testing procedures and a reminder to take the test will be of importance.

\section{CONCLUSIONS}

Clear messages highlighting the benefits of regular testing for family, friends and society in identifying asymptomatic cases are needed. This should be coupled with transparent communication about accuracy of LFTs and how to act on either a positive or a negative result. Concerns about safety, convenience of testing and ability to do tests need to be addressed to ensure successful scaling up of asymptomatic testing.

\section{Author affiliations}

${ }^{1}$ Nuffield Department of Primary Care Health Sciences, University of Oxford, Oxford, UK

${ }^{2}$ University of Oxford Hospitals NHS Foundation Trust, National Institute for Health Research Oxford Biomedical Research Centre, Oxford, UK

${ }^{3}$ Merton College, University of Oxford, Oxford, UK

${ }^{4}$ Wellcome Centre for Integrative Neuroimaging, Wolfson Building, Nuffield Department Clinical Neurosciences, University of Oxford, John Radcliffe Hospital, Oxford, UK

${ }^{5}$ St Hilda's College, University of Oxford, Oxford, UK

${ }^{6}$ Saïd Business School, University of Oxford, Oxford, UK

${ }^{7}$ University of Oxford in Partnership with Public Health England, NIHR Health Protection Research Unit in Healthcare Associated Infections and Antimicrobial Resistance, Oxford, UK

\section{Twitter Rafael Perera @rafaoxford and Brian D Nicholson @BrianDNicholson}

Acknowledgements The authors are grateful to the participants who took part in the study.

Contributors MW designed the study, undertook data collection and analysis, and cowrote the manuscript. ML, CV, JAH, RP, IT, GD, JJL, PT and BDN designed the study and critically appraised the manuscript. JAH designed the study, contributed to the analysis and critically appraised the manuscript. TF and LM designed the study, contributed to the analysis and critically appraised the manuscript. ST-C designed the study, contributed to data analysis and cowrote the manuscript. $\mathrm{RH}$ obtained the funding, designed the study and critically appraised the manuscript.

Funding This study received an internal funding from the University of Oxford (no grant number). MW and ST-C received funding from the EU Horizon 2020 Research and Innovation. ST-C also received funding from the National Institute for Health Research (NIHR) Health Protection Research Unit in Healthcare Associated Infections and Antimicrobial Resistance at the University of Oxford, in partnership with Public Health England. ML and JAH were funded by the NIHR Biomedical Research Centre, Oxford. TF and LM received funding from the NIHR Community Healthcare MedTech and In Vitro Diagnostics Co-operative at Oxford Health NHS Foundation Trust. TF received funding from the NIHR Applied Research Collaboration Oxford and Thames Valley at Oxford Health NHS Foundation Trust. The views expressed are those of the authors and not necessarily those of the NHS, the NIHR or the Department of Health and Social Care. LT acknowledges part support from the NIHR Biomedical Research Centre, Oxford. BDN was funded by an NIHR Academic Clinical Lectureship. FDRH acknowledges part support from the NIHR School for Primary Care Research, the NIHR Collaboration for Leadership in Applied Research in Health and Care Oxford and the NIHR Biomedical Research Centre, Oxford.

Competing interests None declared.

Patient consent for publication Not required.

Ethics approval The study was approved by the University of Oxford Research Ethics Committee in October 2020 (CUREC ethics reference R72896/RE001).

Provenance and peer review Not commissioned; externally peer reviewed.

Data availability statement № data are available.

Supplemental material This content has been supplied by the author(s). It has not been vetted by BMJ Publishing Group Limited (BMJ) and may not have been peer-reviewed. Any opinions or recommendations discussed are solely those of the author(s) and are not endorsed by BMJ. BMJ disclaims all liability and responsibility arising from any reliance placed on the content. Where the content includes any translated material, BMJ does not warrant the accuracy and reliability of the translations (including but not limited to local regulations, clinical guidelines, terminology, drug names and drug dosages), and is not responsible for any error and/or omissions arising from translation and adaptation or otherwise.

Open access This is an open access article distributed in accordance with the Creative Commons Attribution Non Commercial (CC BY-NC 4.0) license, which permits others to distribute, remix, adapt, build upon this work non-commercially, and license their derivative works on different terms, provided the original work is properly cited, appropriate credit is given, any changes made indicated, and the use is non-commercial. See: http://creativecommons.org/licenses/by-nc/4.0/.

\section{ORCID iDs}

Marta Wanat http://orcid.org/0000-0002-0163-1547

Jennifer A Hirst http://orcid.org/0000-0002-8416-2159

Rafael Perera http://orcid.org/0000-0003-2418-2091

Brian D Nicholson http://orcid.org/0000-0003-0661-7362

Sarah Tonkin-Crine http://orcid.org/0000-0003-4470-1151

\section{REFERENCES}

1 World Health Organization. Timeline: WHO's COVID-19 response 2020.

2 World Health Organization. Weekly epidemiological update on COVID-19, 2021. Available: who.int [Accessed 22 Jun 2021].

3 Kashir J, Yaqinuddin A. Loop mediated isothermal amplification (LAMP) assays as a rapid diagnostic for COVID-19. Med Hypotheses 2020;141:109786.

$4 \mathrm{He}$ X, Lau EHY, Wu P, et al. Temporal dynamics in viral shedding and transmissibility of COVID-19. Nat Med 2020;26:672-5.

5 Department of Health and Social Care. Asymptomatic testing to be rolled out across the country starting this week. London, 2021. Available: www.gov.uk

6 European Centre for Disease Prevention and Control. Populationwide testing of SARS-CoV-2: country experiences and potential approaches in the EU/EEA and the United Kingdom. Stockholm: ECDC, 2020. europa.eu

7 Holt E. Slovakia to test all adults for SARS-CoV-2. Lancet 2020;396:1386-7.

8 Raffle AE, Gill M. Mass screening for asymptomatic SARS-CoV-2 infection. BMJ 2021;373:n1058. 
9 Andryukov BG. Six decades of lateral flow immunoassay: from determining metabolic markers to diagnosing COVID-19. AIMS Microbiol 2020;6:280-304.

10 lacobucci G, Coombes R. Covid-19: government plans to spend£ $100 \mathrm{bn}$ on expanding testing to 10 million a day. BMJ 2020.

11 Rudolf F, Kaltenbach H-M, Linnik J, et al. Clinical characterisation of eleven lateral flow assays for detection of COVID-19 antibodies in a population. MedRxiv 2020.

12 World Health Organisation. Target product profiles for priority diagnostics to support response to the COVID-19 pandemic, 2021 Available: who.int

13 UK Government. TFMS: behavioural paper supporting the consensus statement on mass testing, 2020. Available: www.gov.uk [Accessed 27 Aug 2020].

14 UK Government. Multidisciplinary task and finish group on mass testing. consensus statement for SAGE 2020, 2021. Available: publishing.service.gov.uk [Accessed 8 Mar 2021].

15 Torjesen I. Covid-19: how the UK is using lateral flow tests in the pandemic. BMJ 2021;372.

16 Mahase E. Covid-19: UK extends lateral flow test authorisation despite US warning not to use them. BMJ 2021;373:n1582.

17 Mahase E. Covid-19: people are not being warned about pitfalls of mass testing. BMJ 2021.

18 Kmietowicz Z. Covid-19: controversial rapid test policy divides doctors and scientists. BMJ 2021;372:n81.

19 Limb M. Covid-19: use lateral flow tests in schools to find cases, urges independent SAGE. BMJ 2021;372:n75.

20 Eley CV, Sharma A, Lecky DM, et al. Qualitative study to explore the views of general practice staff on the use of point-of-care $C$ reactive protein testing for the management of lower respiratory tract infections in routine general practice in England. BMJ Open 2018;8:e023925.

21 University of Liverpool. Liverpool COVID-19 community testing pilot., 2020. Available: https://www.liverpool.ac.uk/media/livacuk/ coronavirus/Liverpool_Community_Testing_Report.pdf

22 Blake H, Knight H, Jia R, et al. Students' views towards Sars-Cov-2 mass asymptomatic testing, social distancing and self-isolation in a university setting during the COVID-19 pandemic: a qualitative study. Int J Environ Res Public Health 2021;18:4182.

23 Gillam TB, Cole J, Gharbi K, et al. Norwich COVID-19 testing initiative pilot: evaluating the feasibility of asymptomatic testing on a university campus. J Public Health 2021;43:82-8.

24 Innova Medical Group. SARS-CoV-2 antigen rapid qualitative test. Instructions for use 2020 [updated 15 March 2021]. Available: https:// minhalexander.files.wordpress.com/2020/12/instructions-for-useinnova-sars-cov-2-antigen-test-ifu.pdf

25 UK Government. TFMS: behavioural paper supporting the consensus statement on mass testing, 2020. Available: https://www.gov.uk/ government/publications/tfms-behavioural-paper-supporting-theconsensus-statement-on-mass-testing-27-august-2020 [Accessed 27 Aug 2020].

26 NHS. A guide for healthcare staff self-testing for coronavirus using a lateral flow device (LFD), 2020. Available: https://www.england. nhs.uk/coronavirus/wp-content/uploads/sites/52/2020/11/LFD_ NHSStaff_A4_161120_.pdf

27 Fusch PI, Ness LR. Are we there yet? Data saturation in qualitative research. Qual Rep 2015;20:1408.

28 Vindrola-Padros C, Chisnall G, Cooper S, et al. Carrying out rapid qualitative research during a pandemic: emerging lessons from COVID-19. Qual Health Res 2020;30:2192-204.

29 Johnson GA, Vindrola-Padros C. Rapid qualitative research methods during complex health emergencies: a systematic review of the literature. Soc Sci Med 2017;189:63-75.

30 Lecouturier J, Kelly MP, Graham F, et al. Public understanding of COVID-19 antibody testing and test results: a qualitative study conducted in the U.K. early in the pandemic. Soc Sci Med 2021;273:113778.

31 Blake H, Corner J, Cirelli C, et al. Perceptions and experiences of the University of Nottingham pilot sars-cov-2 asymptomatic testing service: a mixed-methods study. Int $J$ Environ Res Public Health 2021;18:188. 